AperTO - Archivio Istituzionale Open Access dell'Università di Torino

\title{
From affective blindsight to emotional consciousness
}

\section{This is the author's manuscript}

Original Citation:

Availability:

This version is available http://hdl.handle.net/2318/1530434

since 2016-11-27T22:59:15Z

Published version:

DOI:10.1016/j.concog.2015.05.007

Terms of use:

Open Access

Anyone can freely access the full text of works made available as "Open Access". Works made available under a Creative Commons license can be used according to the terms and conditions of said license. Use of all other works requires consent of the right holder (author or publisher) if not exempted from copyright protection by the applicable law. 


\title{
From affective blindsight to emotional consciousness
}

\author{
Alessia Celeghin ${ }^{\mathrm{a}, \mathrm{b}, \mathrm{c}}$, Beatrice de Gelder ${ }^{\mathrm{d}}$, Marco Tamietto ${ }^{\mathrm{a}, \mathrm{b}, \mathrm{e}, *}$ \\ ${ }^{a}$ Department of Psychology, University of Torino, Italy \\ ${ }^{\mathrm{b}}$ Department of Medical and Clinical Psychology, CoRPS - Center of Research on Psychology in Somatic Diseases, Tilburg University, The Netherlands \\ ${ }^{\mathrm{c}}$ GCS fMRI, Koelliker Hospital, Torino, Italy \\ ${ }^{\mathrm{d}}$ Department of Cognitive Neuroscience, Maastricht University, The Netherlands \\ e Department of Experimental Psychology, University of Oxford, UK
}

\section{A R T I C L E I N F O}

\section{Article history:}

Received 18 January 2015

Revised 12 May 2015

Accepted 13 May 2015

Available online $\mathrm{xxxx}$

\section{Keywords:}

Cortical blindness

V1

Emotion

Awareness

Amygdala

Superior colliculus

Pulvinar

\begin{abstract}
A B S T R A C T
Following destruction or denervation of the primary visual cortex (V1) cortical blindness ensues. Affective blindsight refers to the uncanny ability of such patients to respond correctly, or above chance level, to visual emotional expressions presented to their blind fields. Fifteen years after its original discovery, affective blindsight still fascinates neuroscientists and philosophers alike, as it offers a unique window on the vestigial properties of our visual system that, though present in the intact brain, tend to be unnoticed or even actively inhibited by conscious processes. Here we review available studies on affective blindsight with the intent to clarify its functional properties, neural bases and theoretical implications. Evidence converges on the role of subcortical structures of old evolutionary origin such as the superior colliculus, the pulvinar and the amygdala in mediating affective blindsight and nonconscious perception of emotions. We conclude that approaching consciousness, and its absence, from the vantage point of emotion processing may uncover important relations between the two phenomena, as consciousness may have evolved as an evolutionary specialization to interact with others and become aware of their social and emotional expressions.
\end{abstract}

(c) 2015 Elsevier Inc. All rights reserved.

\section{Introduction}

Contemporary neuroscience is replete with examples of social and affective processes that occur without or outside conscious experience (de Gelder, de Haan, \& Heywood, 2001; de Gelder, Hortensius, \& Tamietto, 2012; de Gelder \& Tamietto, 2011; Tamietto \& de Gelder, 2010; Vuilleumier, 2005). This evidence has contributed significantly to the burgeoning field of social and affective neuroscience. Possibly, the most insightful and direct access to nonconscious perception of emotional signals is provided by studies on patients with cortical blindness following destruction of the primary visual cortex (V1). In fact, such patients cannot see nor visually acknowledge a visual stimulus presented within the blind area of their visual field (scotoma). The phenomenon, referred to as "affective blindsight", denotes the uncanny ability of such patients to respond correctly, or above chance level, to emotionally salient visual stimuli presented to their blind fields (Anders et al., 2004, 2009; Bertini, Cecere, \& Ladavas, 2013; Cecere, Bertini, Maier, \& Ladavas, 2014; de Gelder \& Hadjikhani, 2006; de Gelder, Morris, \& Dolan, 2005; de Gelder, Pourtois, van Raamsdonk, Vroomen, \& Weiskrantz, 2001; de Gelder, Pourtois, \&

\footnotetext{
* Corresponding author at: Department of Psychology, University of Torino, via Po 14, 10123 Torino, Italy and Department of Medical and Clinical Psychology, Tilburg University, P.O. Box 90153, 5000 LE Tilburg, The Netherlands.
}

E-mail addresses: marco.tamietto@unito.it, M.Tamietto@uvt.nl (M. Tamietto). 
Weiskrantz, 2002; de Gelder, Tamietto, Pegna, \& Van den Stock, 2014; de Gelder, Vroomen, Pourtois, \& Weiskrantz, 1999; Hamm et al., 2003; Heywood \& Kentridge, 2000; Morris, DeGelder, Weiskrantz, \& Dolan, 2001; Pegna, Khateb, Lazeyras, \& Seghier, 2005; Rossion, de Gelder, Pourtois, Guerit, \& Weiskrantz, 2000; Tamietto \& de Gelder, 2008; Tamietto, Pullens, de Gelder, Weiskrantz, \& Goebel, 2012; Tamietto et al., 2009; Van den Stock et al., 2011). As is the case for the original phenomenon of "blindsight” (Pöppel, Held, \& Frost, 1973; Weiskrantz, Warrington, Sanders, \& Marshall, 1974), the "blind” component in affective blindsight refers to the patients' statement of not seeing or consciously perceiving the emotional stimuli, while the "sight" component reflects their residual ability to respond, discriminate or display spontaneous expressive and physiological responses that are appropriate to the specific emotional content of the visual signals they are presented with

Affective blindsight is thus clearly linked to blindsight, but it also poses new questions and has implications for theories of consciousness besides the classic themes tackled in the blindsight literature. In fact, a traditional distinction in the study of consciousness and its neural correlates is between state consciousness and content consciousness (Park \& Tallon-Baudry, 2014; Rees, Kreiman, \& Koch, 2002; Tsuchiya \& Adolphs, 2007). The former applies to the whole organism and refers to vigilance states such as wakefulness, alertness, drowsiness, and coma, whereas the latter denotes that we are aware of the external world or of ourselves. Traditionally, blindsight represents a privileged case-study to tackle the functions of stimulus awareness and, by comparison with conscious vision, to discover the neural signature of content consciousness (Cowey, 2004). As we will discuss in detail later, approaching consciousness, and its absence, from the vantage point of emotion processing may help to reconcile these two aspects of consciousness that have been barely considered within the same framework. In fact, the intersection between emotion and consciousness seems more radical than what was initially envisaged: not only do emotional signals have a privileged access to consciousness, but several subcortical structures that are important for emotion processing partly overlap with structures involved in the maintenance of state consciousness (Craig, 2009; Critchley, 2005; Damasio, 1999; de Gelder \& Tamietto, 2011; Mitchell \& Greening, 2012; Panksepp, 2005; Park \& Tallon-Baudry, 2014; Tamietto \& de Gelder, 2010; Tamietto et al., 2005; Tsuchiya \& Adolphs, 2007; Vuilleumier, 2005). Seen from an evolutionary perspective, this is consistent with the thesis that consciousness evolved in the process, and as a consequence, of the demands of the social and emotional environment (Humphrey, 1983). We would like to argue that this broad affective-evolutionary perspective on consciousness may also be applied to the visual system, at least as a heuristic principle, and may orient the search for specific hypotheses about phylogenetically ancient emotional sensitivity of the visual system of higher organisms.

In this review we will present the studies and evidence accumulating on affective blindsight since its original discovery fifteen years ago, and discuss their theoretical impact for advancing our understanding of the neural mechanisms linking social and emotional vision to consciousness.

\section{The origins}

The first evidence that one patient with destruction of V1 was proficient at discriminating the emotional content of visual stimuli presented to his blind field, without consciously perceiving them, was provided by de Gelder and coworkers in 1999 (de Gelder et al., 1999). The study involved patient GY, a patient with blindness in his right visual field following a lesion to his left V1 and well-documented blindsight (for a detailed description of the patient see: Baseler, Morland, \& Wandell, 1999; Bridge, Thomas, Jbabdi, \& Cowey, 2008; Goebel, Muckli, Zanella, Singer, \& Stoerig, 2001; Tamietto et al., 2010). In May 1997 GY came to our lab in Tilburg, accompanied by Larry Weiskrantz, to give us the opportunity to test some of Paul Bertelson's ideas about the automaticity of ventriloquism and to participate in ongoing studies on the role of visual awareness in audiovisual speech perception. During one of the breaks between these long testing sessions (which ultimately yielded negative results), one of us tried to present facial expressions to GY's blind field and affective blindsight was discovered. The finding was welcomed by healthy skepticism (de Gelder, Vroomen, Pourtois, \& Weiskrantz, 2000; Heywood \& Kentridge, 2000), consistent with the notion that encoding face stimuli is too complex for the visual system deprived of its primary visual cortex (Cowey, 2004; Cowey, 2010). The study consisted of 4 different experiments in which short video clips and still images with happy, sad, angry and fearful facial expressions were presented to the blind as well as intact field of GY. The task required the patient to guess in various forced-choice conditions the emotion conveyed by the stimuli he remained unaware of. Surprisingly, GY was able to discriminate the expressions in his blind field with a reliability exceeding chance level.

Growing evidence in the same period from a number of different areas turned out to be consistent with the results of this first study. For example, neuroimaging studies in healthy subjects had shown amygdala activation along with other subcortical structures receiving direct visual input from the retina, such as the superior colliculus and the pulvinar, in response to facial expressions whose awareness was prevented by visual masking, flash suppression, or filtered in low spatial frequencies (Liddell et al., 2005; Morris, Ohman, \& Dolan, 1998; Morris, Ohman, \& Dolan, 1999; Vuilleumier, Armony, Driver, \& Dolan, 2003; Whalen et al., 1998). Likewise, animal studies using classical conditioning on the auditory system of rodents were demonstrating that the amygdala is able to detect emotionally salient stimuli in the environment and to initiate appropriate responses toward them even before a detailed perceptual analysis was provided by primary sensory cortices (LeDoux, 1996; LeDoux, 2008).

As it often happens with novel and unexpected findings, these first results raised a number of comments and (sometimes dismissive) criticism that have been systematically addressed, and ultimately discarded, in the next years (Cowey, 2004; Cowey, 2010; Heywood \& Kentridge, 2000; Pessoa, 2005). 


\section{What could be mistaken about affective blindsight?}

One first criticism concerned possible basic methodological drawbacks. In fact, the same stimuli projected to the blind field of GY were also previously shown to his seeing field. Because facial expressions of basic emotions are characterized by distinctive and easily detectable visual features (e.g., eyes wide open in fear, or lifted-up lips corners in happiness), correct responses to unseen stimuli may be based on the association and implicit detection of such unique features when the stimuli are first shown to the intact field (Cowey, 2004). This hypothesis was conclusively discarded after affective blindsight was reported in patients presenting a bilateral damage to the occipital cortices (Hamm et al., 2003; Pegna et al., 2005). This condition makes the patient clinically blind over the entire visual field, therefore preventing the use of visual information presented to the seeing field, as the latter simply does not exist.

Another issue concerned the peculiarity of patient GY and, therefore, the generalizability of affective blindsight to other patients with cortical blindness. In fact, GY suffered a lesion strictly confined to his left V1 as a consequence of a traumatic brain injury during a traffic accident that occurred very early in his life, when he was only 7 years old (Barbur, Ruddock, \& Waterfield, 1980; de Gelder et al., 2005; Morris et al., 2001; Sahraie et al., 1997). Thus, it has been proposed, and later on verified with different neuroimaging methods, that considerable post-lesion and experience-dependent plasticity has taken place in GY's brain (Bridge et al., 2008; Tamietto et al., 2012). Although we will discuss the neural underpinnings of affective blindsight in a dedicated section below, it has become increasingly clear that affective blindsight is not such a rare phenomenon, for it has been reported in more than 20 different patients studied by at least five independent research teams in several different countries (Anders et al., 2004, 2009; Bertini et al., 2013; Cecere et al., 2014; de Gelder \& Hadjikhani, 2006; de Gelder et al., 1999, 2001, 2002, 2005, 2014; Hamm et al., 2003; Heywood \& Kentridge, 2000; Morris et al., 2001; Pegna et al., 2005; Rossion et al., 2000; Tamietto \& de Gelder, 2008; Tamietto et al., 2009, 2012; Van den Stock et al., 2011). These patients suffered different lesions to the visual cortex at different ages, but for the large majority in adulthood, and two of them had bilateral cortical blindness. It is therefore reasonable to surmise that affective blindsight, if adequately investigated, can be found in a large population of patients with cortical blindness, devoid of GY's peculiarity.

\section{Methodological issues in the study of affective blindsight}

In the last decade, following significant methodological advances in neuroscience, different measures and methods have been used to study and document affective blindsight. They can be grouped along two orthogonal dimensions. The first relates to the distinction between so-called "direct" and "indirect" methods, whereas the second dimension concerns the differentiation between behavioral measures, on the one hand, and psychophysiological or neurophysiological measures, on the other (Weiskrantz, 1990).

Direct approaches try to circumvent the lack of visual awareness for stimuli presented in the blind field by asking patients to guess which stimulus type, among a limited number of options, has been projected within the blind field. As in the original study, the patients, prompted by consciously perceived auditory stimuli, are forced to guess and to press different buttons for different emotions that may be displayed (de Gelder et al., 1999; Pegna et al., 2005; Van den Stock et al., 2011). Most commonly, direct behavioral measures rely on response accuracy and latency. They have been criticized because they are liable to response strategies or bias and because the (often) dichotomous nature of the measure hardly fits with the more graded nature of our conscious experience (Mitchell \& Greening, 2012; Overgaard, 2011; Pessoa, 2005; Pessoa, Japee, \& Ungerleider, 2005). In fact, analyses of accuracy are typically based on percentage of correct responses and potentially reflect not only patients' sensitivity to a given stimulus attribute, but also their response bias. Therefore, following the original study of Azzopardi and Cowey (1997), scaled reports or post-decision wagering procedures have been introduced, along with signal detection analyses, in the study of affective blindsight or other types of nonconscious emotion perception (Tamietto et al., 2015; Van den Stock et al., 2014). This procedure requires the patient to provide a first response concerning the presence/absence of a stimulus or of one attribute (e.g., its emotional content), and afterward to grade confidence about the first response on a scale. This way, researchers obtain a continuous measure of awareness that can be used to construct receiver operating characteristic curves (ROCs), from which perceptual sensitivity can be derived independently of response criterion, thereby avoiding the problem of response bias.

Indirect methods do not require that patients provide these somewhat counter-intuitive responses to unseen stimuli, but engage them in responding to stimuli projected to their intact fields. By presenting additional stimuli simultaneously to the blind field, evidence of nonconscious processing is inferred if unseen stimuli modulate responses to the normally visible one (Celeghin, Savazzi, Barabas, Bendini, \& Marzi, 2015; Celeghin et al., 2014; Leh, Mullen, \& Ptito, 2006; Leh, Ptito, Schonwiesner, Chakravarty, \& Mullen, 2010; Marzi, Tassinari, Aglioti, \& Lutzemberger, 1986; Tamietto et al., 2010). A classical example of such indirect methods is the redundant target paradigm, in which stimuli are presented either singly to the intact field or paired simultaneously with another stimulus in the blind field. Typically, reaction times (RTs) to the seen stimulus are faster during redundant stimulation than during single presentation to the intact field. This paradigm has been modified for the purposes of studying affective blindsight, thereby presenting pairs of congruent or incongruent emotional expressions to the intact and blind field (Bertini et al., 2013; Cecere et al., 2014; de Gelder et al., 2001; Tamietto \& de Gelder, 2008). A congruency effect has been reported in patient GY, with congruent stimuli yielding faster RTs than incongruent pairs (de Gelder et al., 2001), akin to what was found in healthy observers when visual awareness of one expression in 
a pair was prevented by backward masking (Tamietto \& de Gelder, 2008). Applying a similar design to a group of hemianopic patients without blindsight, Bertini et al. (2013) found instead a boosting effect of unseen fearful expressions over consciously seen faces. Moreover, indirect methods offer valuable insights into mechanisms that mediate the integration between consciously and nonconsciously perceived emotions, both within the visual domain (Bertini et al., 2013; Cecere et al., 2014; de Gelder et al., 2001; Tamietto \& de Gelder, 2008) and, in the case of cross-modal integration, between (consciously perceived) auditory stimuli and (unconsciously perceived) visual expressions (de Gelder et al., 2002, 2005).

Psychophysiological responses have also been used to probe affective blindsight. These measures primarily include eye blink (Hamm et al., 2003), skin conductance (Anders et al., 2004, 2009), pupil dilation and facial electromyography (Tamietto et al., 2009). Eye blink recording samples the startle reflex and gets potentiated after fear conditioning to unseen visual cues (Hamm et al., 2003). Skin conductance and pupil dilation are measures of autonomic arousal induced by sympathetic system activity, and have been reported to increase during passive exposure to facial as well as bodily expressions of emotions projected to the blind field of patients GY and DB (Tamietto et al., 2009). Lastly, electromyography has been used to assess the presence of emotional contagion in patients with affective blindsight (Tamietto et al., 2009). Emotional contagion refers to the spontaneous and automatic tendency to mimic the facial expressions seen in others and, consequently, to converge emotionally (Dimberg, 1982; Dimberg, Thunberg, \& Elmehed, 2000; Hatfield, Cacioppo, \& Rapson, 1994; Tamietto et al., 2009). Such subtle changes in facial musculature are characteristic for different facial expressions of emotions. For instance, happiness involves the contraction of the zygomaticus major that lifts up the corners of the mouth in a smile, while fearful or angry expressions engage the corrugator supercilli in frowning (Dimberg, 1982; Dimberg et al., 2000). Emotional contagion, as measured with electromyography, has been shown in blindsight patients (Tamietto et al., 2009).

The advent of the neuroimaging techniques has boosted investigation of the neural substrate of affective blindsight and, by comparison with seen emotional signals, of the underpinnings of emotional consciousness. We will discuss in a dedicated section the functional and structural neuroanatomy of affective blindsight, while here we only sketch the purposes, major strengths and weaknesses of the different techniques. Functional magnetic resonance imaging (fMRI) is used to characterize the spatial properties of the functional neuroanatomy and the structures activated by unseen emotional stimuli (Anders et al., 2004, 2009; de Gelder \& Hadjikhani, 2006; de Gelder et al., 2005; Morris et al., 2001; Pegna et al., 2005; Van den Stock, Tamietto, Hervais-Adelman, Pegna, \& de Gelder, 2013; Van den Stock et al., 2011, 2014). The first fMRI study on affective blindsight was performed on patient GY by Morris and colleagues and showed that unseen fearful and fear-conditioned facial expressions activate the amygdala, whose response co-varied with activity in the superior colliculus and pulvinar (Morris et al., 2001). The excellent spatial resolution of fMRI, on the order of millimeters, is counterbalanced by its limited temporal resolution, as events occurring during a temporal window of a few seconds are averaged together, thus preventing a fine-grained analysis of the temporal dynamics occurring among the implicated areas. This is a serious limitation when studying emotions, as nonconscious emotional processing takes place within milliseconds after stimulus onset in subcortical brain areas, including the amygdala and structures related to reflex-like motor reactions, and is followed shortly after by later responses engaged in more deliberate responses and in conscious vision (Borgomaneri, Gazzola, \& Avenanti 2014; Borgomaneri, Vitale, Gazzola, \& Avenanti, 2015; Garrido, Barnes, Sahani, \& Dolan, 2012; Garvert, Friston, Dolan, \& Garrido, 2014; Luo, Holroyd, Jones, Hendler, \& Blair, 2007; Maior, Hori, Tomaz, Ono, \& Nishijo, 2010; Nguyen et al., 2014). Because both early nonconscious and later conscious responses take place within the time window of a single volume acquisition in fMRI studies, the different functional values of neural activity in the same structure may be integrated or overridden (Brosch \& Wieser, 2011; Costa et al., 2014; Luo et al., 2010).

This limitation of fMRI has been partially circumvented by the use of methods with a better temporal resolution, such as electroencephalography (EEG) or magnetoencephalography (MEG), which have a temporal resolution on the order of milliseconds, but, on the other hand, have a poor spatial resolution and have been questioned when used to detect neural activity in subcortical structures (Andino, Menendez, Khateb, Landis, \& Pegna, 2009; Cecere et al., 2014; de Gelder et al., 2002; Rossion et al., 2000). Recently, tractography techniques such as diffusor tensor imaging (DTI) have been used to document anatomical connections between structures implicated in blindsight (Bridge et al., 2008; Leh, Johansen-Berg, \& Ptito, 2006) and in affective blindsight (Tamietto et al., 2012), as well as to document post-lesion anatomical plasticity and structural reorganization of fiber connections following lesions to the visual cortex.

Lastly, transcranial magnetic stimulation (TMS) has been used to induce "virtual lesions" to V1, so as to block conscious visual perception and create experimental models of blindsight (Allen, Sumner, \& Chambers, 2014; Lloyd, Abrahamyan, \& Harris, 2013; Railo, Andersson, Kaasinen, Laine, \& Koivisto, 2014; Silvanto, Walsh, \& Cowey, 2009) and affective blindsight (Filmer \& Monsell, 2013; Jolij \& Lamme, 2005). These methods have revealed interesting parallels with the behavioral outcomes reported in affective blindsight. In the future, a better understanding of the actual neurophysiological influences of TMS on the intact brain will provide the basis for a closer comparison with blindsight patients and for a more comprehensive understanding of the systems involved in conscious and nonconscious emotional processing in the intact and damaged brain.

\section{When affective blindsight obtains, when it fails, and why}

Facial expressions effectively communicate emotions during social interactions and, until recently, most investigations of human emotions predominately concentrated on processes associated to conscious or nonconscious perception of faces (Adolphs, 2002; Axelrod, Bar, \& Rees, 2015; Morris et al., 1998, 1999; Whalen et al., 1998). Hence, it is not surprising that 
affective blindsight was initially tested using facial expressions as stimuli. A common theme of inquiry in face perception literature concerns whether the emotional expression is extracted from an analysis of the whole face configuration or rather from the extraction of individual key features in the face (Jack, Garrod, \& Schyns, 2014). This issue is interesting also for affective blindsight, in particular in understanding the minimal visual input sufficient to trigger nonconscious emotion perception. Neuroimaging studies on healthy subjects in whom visual awareness was prevented by backward masking have shown that the amygdala responds to the eye whites, which are particularly informative in conveying fear (Morris, deBonis, \& Dolan, 2002; Whalen et al., 2004). Also, in a recent fMRI study on patient TN with affective blindsight and bilateral cortical blindness, we have reported that the amygdala responds to direct vs. averted gaze (Burra et al., 2013). This seems to suggest that phylogenetically ancient parts of our visual and emotion systems in the brain are tuned to detect this simple signal rather than the whole face configuration, but more investigation is clearly needed here.

Recently, affective blindsight has been tested also for non-facial stimuli, thereby extending evidence of nonconscious emotion processing to other stimulus categories. Bodily expressions of emotions, both static and dynamic, have been the most extensively studied non-facial stimuli. Behavioral, fMRI, and psychophysiological responses from pupil dilation and facial EMG have shown that affective blindsight for bodily expressions may be at least as clearly established as that previously reported for facial expressions and entails a largely overlapping neural system (de Gelder, 2006; de Gelder \& Hadjikhani, 2006; Tamietto et al., 2009; Van den Stock et al., 2011, 2013, 2014).

A certain degree of functional similarity between face and body signals, resulting in their similar role in sustaining nonconscious emotion processing in affective blindsight, challenges theories exclusively concerned with analysis of the specific visual features, and instead suggests an approach that cuts across gross physical stimulus differences, as there exist between facial and bodily expressions, to focus more on the functional properties of visual signals. Under the assumption that the special role of faces is not fixed by their physical properties but by their functional ones, the findings reported above converge with the idea that nonconscious emotion processing in blindsight is not specific for faces, but rather for biologically primitive emotional expressions that are clearly associated with action tendencies and to which we are evolutionary prepared to respond (Tamietto \& de Gelder, 2010).

Our critical perspective on the alleged special status of faces in triggering nonconscious perception seems in keeping with evidence about failure of blindsight when patients are exposed to stimuli that do not clearly share the properties described above. For example, patients with blindsight fail to guess non-emotional facial attributes correctly, such as personal identity or gender (Rossion et al., 2000). Furthermore, facial expressions of complex social emotions, such as arrogance or guilt, also fail to trigger affective blindsight (Celeghin, Adenzato, et al., in preparation). Likewise, complex affective scenes, such as pictures of cockroaches or houses on fire, do not induce affective blindsight, indicating that the patients are unable to guess the emotional content of these scenes (de Gelder et al., 2002). This suggests that the analysis of the emotional content of complex scenes, facial identity or expressions of social emotions may depend critically on conscious visual perception and on the detailed processing of the high spatial frequency information that is typically performed by the cortical visual system.

We have recently addressed this issue in a combined behavioral/fMRI experiment on patients GY and TN (Tamietto et al., in preparation). Fearful and neutral faces were filtered so as to contain only low or only high spatial frequency information. In fact, previous studies have demonstrated that distinct neural pathways in the visual system are differentially sensitive to high and low spatial frequency information in facial expressions (Vuilleumier et al., 2003). High spatial frequency information is predominately relayed to the visual cortex and, from there, to the ventral stream via parvocellular channels with high resolution but relatively slow responses. Conversely, low spatial frequency information is processed by the magnocellular system that encompasses the dorsal stream as well as subcortical structures such as the superior colliculus and the pulvinar that are likely involved in affective blindsight. We reasoned that, if affective blindsight relies on subcortical visual structures and magnocellular channels, then the patients should be able to correctly guess the emotional expressions of faces filtered for displaying only low spatial frequency information, whereas the same expressions filtered in high spatial frequency should knock out the phenomenon. Preliminary behavioral evidence confirms indeed our hypothesis and provides direct support for role of subcortical structures in mediating affective blindsight (Tamietto et al., in preparation).

\section{Which neural systems mediate affective blindsight?}

Starting from the seminal work of Larry Weiskrantz (1956), who first showed that bilateral lesions to the amygdala in monkeys were sufficient to induce a loss of reactivity to emotional visual stimuli typical of the Kluver-Bucy syndrome, the amygdala has been a central structure in the study of emotions. The main pathway for the transmission of visual information from the retina to the amygdala passes through striate and extrastriate cortex along the ventral stream (e.g., LeDoux, 1996). However, earlier studies in rats underlined the role of midbrain structures in providing a rapid but coarse analysis of the affective value of auditory as well as visual stimuli and in relaying such information to the amygdala - even bypassing the primary sensory cortices (Campeau \& Davis, 1995a; Campeau \& Davis, 1995b; Doron \& Ledoux, 1999; Jones \& Burton, 1976; LeDoux, 1996; Linke, De Lima, Schwegler, \& Pape, 1999; Shi \& Davis, 2001).

Reviewing a wide number or neuroimaging and lesion studies investigating nonconscious emotion perception, we concluded that, as far as affective blindsight and sensory unawareness of emotions is concerned, evidence was converging on the existence of a subcortical route from the superior colliculus to the amygdala via the pulvinar nucleus of the thalamus (de Gelder, van Honk, \& Tamietto, 2011; Tamietto \& de Gelder, 2010). Clearly, the existence of such a pathway does not exclude 
other possibilities, nor the role of cortical structures in other forms of conscious or nonconscious emotion perception (Pessoa \& Adolphs, 2011). We will first consider functional and anatomical evidence and discuss in a separate session the timing issue and processing speed in the subcortical pathway to the amygdala.

\subsection{Functional and structural neuroanatomy}

Neuroimaging data on healthy subjects in whom nonconscious perception of emotions had been induced by experimental manipulations such as visual masking, flash suppression, or spatial filtering have revealed that the superior colliculus, pulvinar and amygdala constitute a functional network that shows increased, positive covariation of activity in response to nonconsciously perceived fearful facial expressions (Liddell et al., 2005; Morris et al., 1999; Vuilleumier et al., 2003; Whalen et al., 1998, 2004; Williams et al., 2006). By contrast, the major cortical pathway relaying visual input to the amygdala does not show substantial activity and functional connectivity under the same conditions but does so during conscious perception of emotional stimuli (Pasley, Mayes, \& Schultz, 2004; Williams et al., 2006). Not surprisingly, similar findings have been reported in patients with affective blindsight in response to unseen facial and bodily expressions and indicate that a functional subcortical pathway to the amygdala is engaged during nonconscious emotion perception (de Gelder et al., 2005; Morris et al., 2001; Pegna et al., 2005; Van den Stock et al., 2011, 2013).

The functional role of the superior colliculus and pulvinar in processing emotional expressions has received independent support from recent single cell recordings in monkeys (macaca fuscata) (Nguyen et al., 2014). In fact, a subpopulation of superior colliculus' neurons responds to face and face-like visual stimuli, and its response properties are not influenced when by low spatial frequency filtering of the images. Moreover, neural response magnitude and latency to face stimuli in the superior colliculus significantly correlates with those in the pulvinar. Another cell recording study from the same group showed that monkey pulvinar neurons display differential activity to specific emotional expressions (Maior et al., 2010). Lastly, there is evidence about direct involvement of the superior colliculus in approach and defensive behavior in rodents (Comoli et al., 2012; Liang \& et al., 2015) as well as humans (Billington, Wilkie, Field, \& Wann, 2011).

Behavioral studies on patients with selective pulvinar lesions also demonstrated that such patients show a marked impairment in fear recognition for stimuli presented to the contralesional field when the damage involves the medial pulvinar, thereby indicating the medial pulvinar as a point of convergence for both subcortical and cortical systems implicated in fear perception (Ward, Calder, Parker, \& Arend, 2007; Ward, Danziger, \& Bamford, 2005). Furthermore, in a recent collaborative study with the group of Alain Ptito, we tested affective blindsight in two patients with hemispherectomy, DR and SE, who have previously shown blindsight for non-emotional stimuli during redundant target experiments (Celeghin, Diano, et al., in preparation). In such patients, the entire cortical mantel of one hemisphere has been removed for clinical purposes, including the amygdala and large portions of the pulvinar, and leaving only the superior colliculus entirely intact. Using indirect methods we have been able to show nonconscious processing of unseen emotional stimuli in such patients. These initial findings would suggest not only that the superior colliculus is involved in affective blindsight, but also, and more conclusively, that the cortex is not strictly necessary for affective blindsight to occur; although cortical contribution can clearly sustain several aspects of the phenomenon, such facial mimicry or affective feelings (Anders et al., 2004, 2009; Tamietto et al., 2009).

Assuming the role of a functional pathway, are these structures also anatomically connected, thereby forming a structural pathway? While tracer studies have demonstrated the existence in birds and rodents of anatomical connections between the superior colliculus, pulvinar and amygdala, similar evidence in primates was lacking until recently (Pessoa, 2005; Pessoa \& Adolphs, 2011). However, Day-Brown, Wei, Chomsung, Petry, and Bickford (2010) have shown that in the tree shrew (tupaia belangeri), a prototypical primate for the anatomical organization of its visual system, projections to the lateral amygdala originate also from the dorsal pulvinar that receives visual input from the superior colliculus, thereby forming a disynaptic pathway to the amygdala. The authors suggested that this pathway potentially relays non-topographic visual information from the SC to the amygdala, its functional role being that of alerting the animal to potentially dangerous signals (Day-Brown et al., 2010).

In an attempt to verify whether such anatomical connections also exist in the human brain, we used DTI to characterize in vivo the connectivity between the superior colliculus, pulvinar and amygdala in normal observers and its changes in blindsight patient GY (Tamietto et al., 2012). We found fiber connections between pulvinar and amygdala and also between superior colliculus and amygdala via the pulvinar in the healthy observer as well as in the patient GY. The destruction of the visual cortex led to qualitative and quantitative modifications along the pathways connecting these three structures, and the changes were confined to the patient's damaged hemisphere, thereby strongly supporting the notion that the subcortical route conveys visual information critical for sustaining affective blindsight. A recent tractography study from an independent group used a different tractography method in twenty healthy subjects as well as in eight monkeys (macaca mulatta) to trace possible direct connections between colliculus, pulvinar and amygdala (Rafal et al., submitted). Results in humans were closely comparable to our previous findings and, in additions, the study provided the first anatomical evidence of direct connections in the monkey brain.

\subsection{Timing and speed of processing along the subcortical pathway}

Another way to tackle the issue of whether the subcortical pathway to the amygdala plays a crucial role in affective blindsight is to consider the timing of responses in the subcortical structures involved, and to compare such responses to those 
generated in early cortical areas during conscious emotion perception. Historically, the emphasis on the advantage in speed of processing for the subcortical over cortical route in emotion processing originated from the studies of LeDoux testing fear conditioning in the auditory system of rodents, and has been transferred by analogy to nonconscious visual processing of emotions in humans (LeDoux, 1996; Pessoa \& Adolphs, 2011). However, the rodent auditory system and the human visual system differ in major ways, and it is thus wise to move beyond analogy and metaphors. Admittedly, due to current methodological limitations of EEG and MEG in sampling neural activity in deep structures, evaluation and comparison of processing speed in cortical and subcortical structures of the human brain is still an open issue. Data from cell recordings in monkeys thus represent a more solid starting ground.

For example, the same study recording activity in the monkeys' superior colliculus in response to face and face-like stimuli reported that superior colliculus neurons could encode face-like stimuli within the early $25 \mathrm{~ms}$ post stimulus onset, albeit stimulus encoding further develops in the next 75 ms (Nguyen et al., 2014). For comparison, consider that the average response latency in macaques' $\mathrm{V} 1$ ranges from 60 to $80 \mathrm{~ms}$ and the response to face stimuli occur around 100-150 ms post stimulus onset (Lamme \& Roelfsema, 2000; Schmolesky et al., 1998). According to the authors, superior colliculus neurons filter face-like patterns with short latency to enable rapid processing of coarse facial information, while stimulus categorization develops in later processing phases through feedback from upstream areas. Likewise, the earliest response latency in visually responsive pulvinar neurons that differentiates between facial expressions were shorter than $100 \mathrm{~ms}$, but ranged widely, i.e. up to $300 \mathrm{~ms}$ (Maior et al., 2010). This distribution of response latency suggests that the pulvinar mediates intracortical connections as well as faster subcortical feedforward connections to the amygdala. Lastly, response latency in the monkey amygdala selective for faces and facial expressions ranges from 100 to $150 \mathrm{~ms}$ (Gothard, Battaglia, Erickson, Spitler, \& Amaral, 2007; Kuraoka \& Nakamura, 2007).

Human studies using EEG and MEG have provided mixed results. One study combining MEG and MRI methods reported early event-related synchronization in the pulvinar at about $10-20 \mathrm{~ms}$ after presentation of fearful faces, followed by event-related synchronization in the amygdala at $20-30 \mathrm{~ms}$ after onset, whereas synchronization in the striate cortex occurred 40-50 ms after stimulus onset (Luo et al., 2007). Another study revealed dissociation between rapid amygdala responses to automatic fearful face processing and later responses that interacted with voluntary attention. In fact, a rapid increase in gamma band activity in response to threatening faces $(30-60 \mathrm{~ms})$ was shown to be independent of task load, while a significant interaction of emotion with attention manipulation was seen at later latencies (280-340 ms) (Luo et al., 2010). Finally, two recent MEG studies used dynamic causal modeling (DCM) to test the explanatory power of the subcortical route to amygdala versus a model predicting only cortical mediation over amygdala activity. Early brain activity was better explained by a model including a subcortical pathway, whereas at longer latencies both models had comparable explanatory power (Garrido et al., 2012; Garvert et al., 2014).

Besides data from specific studies, a more theoretical consideration is important when discussing the alleged speed of the subcortical route for nonconscious emotion perception. In fact, the debate often concentrates on the timing of neuronal firing in one or another brain area following presentation of emotional stimuli. However, the crucial aspect, in evolutionary terms, is not which brain area starts firing first, but which neural pathway supports quicker implementation of the instrumental and expressive affective programs typically associated to emotional responses (de Gelder et al., 2011). There is indeed no obvious or direct relation between the latency of neural activity in different brain areas and the latency required by that activity to trigger a behavioral response. The only study that investigated spontaneous facial reactions to emotional stimuli in patients with blindsight found that expressive reactions are triggered faster when the emotional stimulus is projected to the blind field and likely processed by the subcortical pathway to the amygdala (Tamietto et al., 2009). Moreover, it should be bared in mind that an appropriate instrumental response may also include the absence of over behavior, such as during freezing or "attentive immobility" when threat is perceived at a distance. A common finding in behavioral studies on humans is the slowing down of RTs for negative stimuli, such as fearful or angry expressions (e.g., Tamietto, Latini Corazzini, de Gelder, \& Geminiani, 2006). It has been suggested that slower response latencies to threatening stimuli are induced by amygdala activity and reflect immobility, analogous to freezing in animals (Sagaspe, Schwartz, \& Vuilleumier, 2011).

\section{From integration of conscious and nonconscious emotion perception to emotional consciousness}

Is the processing of unseen emotional signals confined to the nonconscious domain, or may it influence our conscious perception of, and reactions to, the seen world? Several studies on affective blindsight have used indirect methods to investigate possible on-line interactions between consciously and nonconsciously perceived emotions, as well as the influence exerted by the former over ongoing recognition of seen stimuli (Bertini et al., 2013; Cecere et al., 2014; de Gelder et al., 2001; Tamietto \& de Gelder, 2008). Unimodal (visual/visual) and cross-modal interactions (visual/auditory) between consciously and nonconsciously perceived emotional stimuli have been reported to occur in affective blindsight patients. For example, in patient GY conscious recognition of facial expressions is speeded up if another face showing the same expression is presented to the blind field (de Gelder et al., 2001, 2005). Interestingly, preliminary findings indicate that an unseen stimulus may also bias responses to a seen stimulus with very different physical properties, such as a facial and a bodily expression, providing they to convey a congruent emotional meaning (Tamietto, Geminiani, \& de Gelder, 2006). The same type of interaction has also been studied in multisensory conditions with unseen visual stimuli modulating the auditory processing 
of audiovisual stimulus pairs. Presenting an incongruent facial expression to the blind field indeed biases the judgment of the emotional prosody of a sentence fragment (de Gelder et al., 2002, 2005).

Partially different results have been recently reported in two studies on hemianopic patients who did not show behavioral evidence of blindsight (Bertini et al., 2013; Cecere et al., 2014). Such patients exhibited faster responses to seen happy faces in their intact visual field when unseen fearful faces were concurrently presented to their blind field. Thus, in the present case there was not a congruency effect between seen and unseen expressions, as reported in patient GY, but a more generalized boosting effect of unseen fearful expressions. This outcome has been suggested to reflect early activation of the subcortical pathway for implicit fear perception. In a follow up study with EEG, the amplitude of the N170 elicited by seen happy faces selectively increased when an unseen fearful face was concurrently presented to the blind field of right hemianopic patients (Cecere et al., 2014). These interesting findings are not necessarily in contrast with the previous reports on patient GY. As it happens, they suggest an intriguing difference between patients displaying affective as well as "non-affective" blindsight, such as GY, and hemianopic patients who do not have blindsight but nevertheless are influenced by fearful expressions in the blind field. Indeed, both phenomena may be mediated by a visual system deprived of its primary visual cortex input but with intact subcortical structures, and thus share propensities to nonconscious emotion processing. However, the possibly different role of spared extrastriate visual areas may explain the different effects of the interaction between seen and unseen affective stimuli. Therefore, patients such as GY, in whom blindsight for emotions as well as for various non-emotional visual properties converge, can tell us something about the interaction of the two phenomena, whereas patients with hemianopia without blindsight can reveal possible dissociations. This issue clearly deserves further study and a direct comparison or the two populations with identical stimuli, settings and experimental design.

As we noted already, affective blindsight offers a unique window on the vestigial properties implemented in subcortical structures to encode and promote reactions in response to emotional stimuli. These properties are present in the intact brain as well, but tend to be overruled or inhibited by cortical processes. In fact, physiological responses in healthy observers tend to be faster or stronger when the eliciting emotional stimuli are not consciously processed (Williams et al., 2004). Likewise, spontaneous facial reactions occur faster for unseen than seen facial or bodily expressions in blindsight patients (Tamietto et al., 2009), and peripheral arousal, as indexed by pupil dilation, is enhanced for unaware stimuli in both, patients with affective blindsight or patients with neglect (Tamietto et al., 2015). This enhanced influence of nonconscious emotions is in line with evidence that cortical activity may exert an inhibitory modulation over subcortical areas (Bush \& Sejnowski, 1996). Absence or reduction of such inhibition during nonconscious perception of emotional stimuli could also explain the apparently paradoxical finding that subcortical activity can be enhanced during nonconscious compared to conscious perception of emotional stimuli in healthy subjects (Anderson, Christoff, Panitz, De Rosa, \& Gabrieli, 2003; Williams, Morris, McGlone, Abbott, \& Mattingley, 2004). Likewise, conscious perception of the eliciting stimulus can overweight subjective affective experience in response to an aversively conditioned stimulus, and the decoupling between phenomenal affective experience and actual physiological changes is associated with increased activity in the ventro-lateral prefrontal cortex (Anders et al., 2004, 2009).

The basic physiological reactions triggered by unseen emotional stimuli involve the moment-to-moment mapping of our bodily states and interoceptive information crucial for homeostatic regulation. Because homeostatic processes provide the sense of invariance that accompanies every subjective experience, they constitute a neurobiological mechanism for the invariance of the sense of self and the continuity of our first-person experience of the world (Damasio, 1999; Park \& Tallon-Baudry, 2014; Tsuchiya \& Adolphs, 2007). Accordingly, basic aspects of the physiological reactions to emotional stimuli, including reactions to stimuli that are not consciously perceived, overlap with physiological responses related to corrections of homeostatic imbalance and thought to be necessary for the general level of consciousness (Damasio, 1999; Damasio \& Carvalho, 2013; Zeman, 2001). It is no coincidence that these emotional responses are controlled by neural structures in the brainstem that also control the level of consciousness. Therefore, even when we remain unaware of the external determinant of an emotional response, such that the eliciting stimulus does not become a content of our conscious visual experience, the chain of physiological reactions it triggers nevertheless contributes to modulate our state of vigilance and behavior, which are constitutive components of our state consciousness.

But can the bodily changes triggered by unseen emotional stimuli be themselves experienced consciously as feelings? Normally, we are not aware of such bodily responses if they cannot be linked to conscious representations of their external (e.g., an angry expression or a sudden noise) or internal causes (e.g., our thoughts). Therefore, both the external determinants as well as the associated physiological responses can fail to become the content of our conscious experience. As we discussed already, passive exposure to unseen facial or bodily expressions in blindsight or healthy participants can induce subtle changes in the observer's facial muscles or arousal responses the subject remains unaware of (Dimberg et al., 2000; Tamietto et al., 2009). Nevertheless, our behavior can still be modulated by unseen emotional signals seemingly without any accompanying conscious feeling. For example, consumption behaviors or preference judgments can be influenced by exposure to masked facial expressions, despite subjective feelings remain unaltered (Winkielman \& Berridge, 2004). Also, a patient with profound impairment in taste perception (ageusia) due to bilateral damage involving the gustatory cortex, could not consciously distinguish between sweet or saline beverages and did not report any associated conscious feeling, but still consistently preferred the sugar solution when provided with the opportunity to choose (Adolphs, Tranel, Koenigs, \& Damasio, 2005).

It is conceivable, however, that in specific situations or clinical conditions, such as alexithymia, pathological anxiety or depression we can become aware of our physiological changes without any conscious representation of their underlying 
causes. Adopting the terminology used in the introduction, bodily changes can be felt and become a content of our conscious experience, while the triggering events do not. One study on patients with cortical blindness due to V1 lesions has shown that the presentation of an unseen stimulus previously paired with an aversive event enhances eye-blink startle reflex, and this enhancement corresponded to the reported level of negative emotional feelings (Anders et al., 2004). Yet it is unknown whether such feelings can be induced by unseen stimuli intrinsically emotional, like facial expressions, and whether they can influence the above-chance discrimination between different unseen emotional stimuli reported in patients with affective blindsight.

Fifteen years of investigation on affective blindsight have contributed to our understanding of the neurobiological basis and of psychophysiological responses to emotional visual stimuli not perceived consciously. This provides important new directions for our understanding of the visual system and of emotion mechanisms. Even more remarkably, affective blindsight offers a unique and valuable testing ground to conjointly study and to link within an integrated theoretical framework emotion and consciousness.

\section{Conflict of interest}

The authors declare no competing financial interests.

\section{Acknowledgments}

Marco Tamietto and Alessia Celeghin are supported by a "Vidi" Grant from the Netherlands Organization for Scientific Research (NWO) (Grant 452-11-015) and by a FIRB - Futuro in Ricerca 2012 - Grant from the Italian Ministry of Education University and Research (MIUR) (Grant RBFR12F0BD). Beatrice de Gelder is supported by the European Research Council under the European Union's Seventh Framework Programme, ERC Advanced Grant.

\section{References}

Adolphs, R. (2002). Neural systems for recognizing emotion. Current Opinion in Neurobiology, 12(2), $169-177$.

Adolphs, R., Tranel, D., Koenigs, M., \& Damasio, A. R. (2005). Preferring one taste over another without recognizing either. Nature Neuroscience, 8(7), $860-861$.

Allen, C. P., Sumner, P., \& Chambers, C. D. (2014). The timing and neuroanatomy of conscious vision as revealed by TMS-induced blindsight. Journal of Cognitive Neuroscience, 26(7), 1507-1518.

Anders, S., Birbaumer, N., Sadowski, B., Erb, M., Mader, I., Grodd, W., et al (2004). Parietal somatosensory association cortex mediates affective blindsight. Nature Neuroscience, 7(4), 339-340.

Anders, S., Eippert, F., Wiens, S., Birbaumer, N., Lotze, M., \& Wildgruber, D. (2009). When seeing outweighs feeling: A role for prefrontal cortex in passive control of negative affect in blindsight. Brain, 132(Pt 11), 3021-3031.

Anderson, A. K., Christoff, K., Panitz, D., De Rosa, E., \& Gabrieli, J. D. (2003). Neural correlates of the automatic processing of threat facial signals. Journal of Neuroscience, 23, 5627-5633.

Andino, S. L., Menendez, R. G., Khateb, A., Landis, T., \& Pegna, A. J. (2009). Electrophysiological correlates of affective blindsight. Neuroimage, 44(2), 581-589. Axelrod, V., Bar, M., \& Rees, G. (2015). Exploring the unconscious using faces. Trends in Cognitive Sciences, 19(1), 35-45.

Azzopardi, P., \& Cowey, A. (1997). Is blindsight like normal, near-threshold vision? Proceedings of the National Academy of Sciences, USA, 94(25), $14190-14194$

Barbur, J. L., Ruddock, K. H., \& Waterfield, V. A. (1980). Human visual responses in the absence of the geniculo- calcarine projection. Brain, 103, 905-928.

Baseler, H. A., Morland, A. B., \& Wandell, B. A. (1999). Topographic organization of human visual areas in the absence of input from primary cortex. Journal of Neuroscience, 19(7), 2619-2627.

Bertini, C., Cecere, R., \& Ladavas, E. (2013). I am blind, but I “see” fear. Cortex, 49(4), 985-993.

Billington, J., Wilkie, R. M., Field, D. T., \& Wann, J. P. (2011). Neural processing of imminent collision in humans. Proceedings of the Royal Society B: Biological Sciences, 278, 1476-1481.

Borgomaneri, S., Gazzola, V., \& Avenanti, A. (2014). Transcranial magnetic stimulation reveals two functionally distinct stages of motor cortex involvement during perception of emotional body language. Brain Structure and Function. http://dx.doi.org/10.1007/s00429-014-0825-6 [Epub ahead of print].

Borgomaneri, S., Vitale, F., Gazzola, V., \& Avenanti, A. (2015). Seeing fearful body language rapidly freezes the observer's motor cortex. Cortex, 65, $232-245$.

Bridge, H., Thomas, O., Jbabdi, S., \& Cowey, A. (2008). Changes in connectivity after visual cortical brain damage underlie altered visual function. Brain, 131(Pt 6), 1433-1444.

Brosch, T., \& Wieser, M. J. (2011). The (non)automaticity of amygdala responses to threat: On the issue of fast signals and slow measures. Journal of Neuroscience, 31(41), 14451-14452.

Burra, N., Hervais-Adelman, A., Kerzel, D., Tamietto, M., de Gelder, B., \& Pegna, A. J. (2013). Amygdala activation for eye contact despite complete cortical blindness. Journal of Neuroscience, 33(25), 10483-10489.

Bush, P., \& Sejnowski, T. (1996). Inhibition synchronizes sparsely connected cortical neurons within and between columns in realistic network models. Journal of Computational Neuroscience, 3, 91-110.

Campeau, S., \& Davis, M. (1995a). Involvement of subcortical and cortical afferents to the lateral nucleus of the amygdala in fear conditioning measured with fear-potentiated startle in rats trained concurrently with auditory and visual conditioned stimuli. Journal of Neuroscience, 15 (3 Pt 2), $2312-2327$.

Campeau, S., \& Davis, M. (1995b). Involvement of the central nucleus and basolateral complex of the amygdala in fear conditioning measured with fearpotentiated startle in rats trained concurrently with auditory and visual conditioned stimuli. Journal of Neuroscience, 15(3 Pt 2), $2301-2311$.

Cecere, R., Bertini, C., Maier, M. E., \& Ladavas, E. (2014). Unseen fearful faces influence face encoding: Evidence from ERPs in hemianopic patients. Journal of Cognitive Neuroscience, 26(11), 2564-2577.

Celeghin, A., Barabas, M., Mancini, F., Bendini, M., Pedrotti, E., Prior, M., et al (2014). Speeded manual responses to unseen visual stimuli in hemianopic patients: What kind of blindsight? Consciousness and Cognition, 32, 6-14.

Celeghin, A., Adenzato, M., Mosso, C. O., Weiskrantz, L., de Gelder, B., \& Tamietto, M. (in preparation). Affective blindsight for basic but not for social emotions.

Celeghin, A., Diano, M., Georgy, L., de Gelder, B., Ptito, A., \& Tamietto, M. (in preparation). Affective blindsight in patients with hemispherectomy.

Celeghin, A., Savazzi, S., Barabas, M., Bendini, M., \& Marzi, C. A. (2015). Blindsight is sensitive to stimulus numerosity and configuration: Evidence from the redundant signal effect. Experimental Brain Research, 233(5), 1617-1623. 
Comoli, E., Das Neves Favaro, P., Vautrelle, N., Leriche, M., Overton, P. G., \& Redgrave, P. (2012). Segregated anatomical input to sub-regions of the rodent superior colliculus associated with approach and defense. Frontiers in Neuroanatomy, 6, 9. http://dx.doi.org/10.3389/fnana.2012.00009.

Costa, T., Cauda, F., Crini, M., Tatu, M. K., Celeghin, A., de Gelder, B., et al (2014). Temporal and spatial neural dynamics in the perception of basic emotions from complex scenes. Social Cognitive and Affective Neuroscience, 9(11), 1690-1703.

Cowey, A. (2004). The 30th Sir Frederick Bartlett lecture. Fact, artefact, and myth about blindsight. Quarterly Journal of Experimental Psychology A, 57(4), 577-609.

Cowey, A. (2010). The blindsight saga. Experimental Brain Research, 200(1), 3-24.

Craig, A. D. (2009). How do you feel-now? The anterior insula and human awareness. Nature Reviews Neuroscience, 10(1), 59-70.

Critchley, H. D. (2005). Neural mechanisms of autonomic, affective, and cognitive integration. Journal of Comparative Neurology, 493(1), 154-166.

Damasio, A. R. (1999). The feeling of what happens: Body and emotion in the making of consciousness. New York: Harcourt Brace and Company.

Damasio, A., \& Carvalho, G. B. (2013). The nature of feelings: Evolutionary and neurobiological origins. Nature Reviews Neuroscience, 14(2), 143-152.

Day-Brown, J. D., Wei, H., Chomsung, R. D., Petry, H. M., \& Bickford, M. E. (2010). Pulvinar projections to the striatum and amygdala in the tree shrew. Frontiers in Neuroanatomy, 4, 143.

de Gelder, B. (2006). Towards the neurobiology of emotional body language. Nature Reviews Neuroscience, 7(3), 242-249.

de Gelder, B., de Haan, E., \& Heywood, C. (2001). Out of mind. Varieties of unconscious processes. Oxford: Oxford University Press.

de Gelder, B., \& Hadjikhani, N. (2006). Non-conscious recognition of emotional body language. NeuroReport, 17(6), 583-586.

de Gelder, B., Hortensius, R., \& Tamietto, M. (2012). Attention and awareness each influence amygdala activity for dynamic bodily expressions - A short review. Frontiers in Integrative Neuroscience, 6, 54.

de Gelder, B., Morris, J. S., \& Dolan, R. J. (2005). Unconscious fear influences emotional awareness of faces and voices. Proceedings of the National Academy of Sciences, USA, 102(51), 18682-18687.

de Gelder, B., Pourtois, G., van Raamsdonk, M., Vroomen, J., \& Weiskrantz, L. (2001). Unseen stimuli modulate conscious visual experience: Evidence from inter-hemispheric summation. NeuroReport, 12(2), 385-391.

de Gelder, B., Pourtois, G., \& Weiskrantz, L. (2002). Fear recognition in the voice is modulated by unconsciously recognized facial expressions but not by unconsciously recognized affective pictures. Proceedings of the National Academy of Sciences, USA, 99(6), 4121-4126.

de Gelder, B., \& Tamietto, M. (2011). Faces, bodies, social vision as agent vision and social consciousness. In R. Adams, J. N. Ambady, K. Nakayama, \& S. Shimojo (Eds.), The science of social vision (pp. 51-74). New York: Oxford University Press.

de Gelder, B., Tamietto, M., Pegna, A. J., \& Van den Stock, J. (2014). Visual imagery influences brain responses to visual stimulation in bilateral cortical blindness. Cortex.

de Gelder, B., van Honk, J., \& Tamietto, M. (2011). Emotion in the brain: Of low roads, high roads and roads less travelled. Nature Reviews Neuroscience, 12(7), 425 [author reply 425].

de Gelder, B., Vroomen, J., Pourtois, G., \& Weiskrantz, L. (1999). Non-conscious recognition of affect in the absence of striate cortex. NeuroReport, 10(18), 3759-3763.

de Gelder, B., Vroomen, J., Pourtois, G., \& Weiskrantz, L. (2000). Affective blindsight: Are we blindly led by emotions? Response to Heywood and Kentridge (2000). Trends in Cognitive Sciences, 4(4), 126-127.

Dimberg, U. (1982). Facial reactions to facial expressions. Psychophysiology, 19(6), 643-647.

Dimberg, U., Thunberg, M., \& Elmehed, K. (2000). Unconscious facial reactions to emotional facial expressions. Psychological Science, 11(1), 86-89.

Doron, N. N., \& Ledoux, J. E. (1999). Organization of projections to the lateral amygdala from auditory and visual areas of the thalamus in the rat. The Journal of Comparative Neurology, 412(3), 383-409.

Filmer, H. L., \& Monsell, S. (2013). TMS to V1 spares discrimination of emotive relative to neutral body postures. Neuropsychologia, 51(13), 2485-2491.

Garrido, M. I., Barnes, G. R., Sahani, M., \& Dolan, R. J. (2012). Functional evidence for a dual route to amygdala. Current Biology. http://dx.doi.org/10.1016/ j.cub.2011.1011.1056.

Garvert, M. M., Friston, K. J., Dolan, R. J., \& Garrido, M. I. (2014). Subcortical amygdala pathways enable rapid face processing. Neuroimage, 102(Pt 2), 309-316.

Goebel, R., Muckli, L., Zanella, F. E., Singer, W., \& Stoerig, P. (2001). Sustained extrastriate cortical activation without visual awareness revealed by fMR studies of hemianopic patients. Vision Research, 41(10-11), 1459-1474.

Gothard, K. M., Battaglia, F. P., Erickson, C. A., Spitler, K. M., \& Amaral, D. G. (2007). Neural responses to facial expression and face identity in the monkey amygdala. Journal of Neurophysiology, 97(2), 1671-1683.

Hamm, A. O., Weike, A. I., Schupp, H. T., Treig, T., Dressel, A., \& Kessler, C. (2003). Affective blindsight: Intact fear conditioning to a visual cue in a cortically blind patient. Brain, 126(Pt 2), 267-275.

Hatfield, H., Cacioppo, J. T., \& Rapson, R. L. (1994). Emotional contagion. Cambridge, MA: Cambridge University Press.

Heywood, C. A., \& Kentridge, R. W. (2000). Affective blindsight? Trends in Cognitive Sciences, 4(4), 125-126.

Humphrey, N. K. (1983). Consciousness regained: Chapters in the development of mind. Oxford: Oxford University Press.

Jack, R. E., Garrod, O. G., \& Schyns, P. G. (2014). Dynamic facial expressions of emotion transmit an evolving hierarchy of signals over time. Current Biology, 24(2), 187-192.

Jolij, J., \& Lamme, V. A. (2005). Repression of unconscious information by conscious processing: Evidence from affective blindsight induced by transcranial magnetic stimulation. Proceedings of the National Academy of Sciences, USA, 102(30), 10747-10751.

Jones, E. G., \& Burton, H. (1976). A projection from the medial pulvinar to the amygdala in primates. Brain Research, 104(1), 142-147.

Kuraoka, K., \& Nakamura, K. (2007). Responses of single neurons in monkey amygdala to facial and vocal emotions. Journal of Neurophysiology, 97(2), 1379-1387.

Lamme, V. A., \& Roelfsema, P. R. (2000). The distinct modes of vision offered by feedforward and recurrent processing. Trends in Neurosciences, 23(11), $571-579$.

LeDoux, J. E. (1996). The emotional brain. New York: Simon \& Shuster.

LeDoux, J. E. (2008). Unconscious processing of fear-arousing events. In L. Weiskrantz \& M. S. Davies (Eds.), Frontiers of consciousness (pp. 76-86). Oxford: Oxford University Press.

Leh, S. E., Johansen-Berg, H., \& Ptito, A. (2006a). Unconscious vision: new insights into the neuronal correlate of blindsight using diffusion tractography Brain, 129(Pt 7), 1822-1832.

Leh, S. E., Mullen, K. T., \& Ptito, A. (2006b). Absence of S-cone input in human blindsight following hemispherectomy. European Journal of Neuroscience, 24(10), 2954-2960.

Leh, S. E., Ptito, A., Schonwiesner, M., Chakravarty, M. M., \& Mullen, K. T. (2010). Blindsight mediated by an S-cone-independent collicular pathway: An fMR study in hemispherectomized subjects. Journal of Cognitive Neuroscience, 22(4), 670-682.

Liang et al (2015). Sensory cortical control of a visually induced arrest behavior via corticotectal projections. Neuron. http://dx.doi.org/10.1016/ j.neuron.2015.03.048

Liddell, B. J., Brown, K. J., Kemp, A. H., Barton, M. J., Das, P., Peduto, A., et al (2005). A direct brainstem-amygdala-cortical 'alarm' system for subliminal signals of fear. Neuroimage, 24(1), 235-243.

Linke, R., De Lima, A. D., Schwegler, H., \& Pape, H. C. (1999). Direct synaptic connections of axons from superior colliculus with identified thalamoamygdaloid projection neurons in the rat: Possible substrates of a subcortical visual pathway to the amygdala. The Journal of Comparative Neurology, 403(2), 158-170.

Lloyd, D. A., Abrahamyan, A., \& Harris, J. A. (2013). Brain-stimulation induced blindsight: Unconscious vision or response bias? PLoS ONE, 8(12), e82828. 
Luo, Q., Holroyd, T., Jones, M., Hendler, T., \& Blair, J. (2007). Neural dynamics for facial threat processing as revealed by gamma band synchronization using MEG. Neuroimage, 34(2), 839-847.

Luo, Q., Holroyd, T., Majestic, C., Cheng, X., Schechter, J., \& Blair, R. J. (2010). Emotional automaticity is a matter of timing. Journal of Neuroscience, 30(17), $5825-5829$.

Maior, R. S., Hori, E., Tomaz, C., Ono, T., \& Nishijo, H. (2010). The monkey pulvinar neurons differentially respond to emotional expressions of human faces. Behavioural Brain Research, 215(1), 129-135.

Marzi, C. A., Tassinari, G., Aglioti, S., \& Lutzemberger, L. (1986). Spatial summation across the vertical meridian in hemianopics: A test of blindsight. Neuropsychologia, 24(6), 749-758.

Mitchell, D. G., \& Greening, S. G. (2012). Conscious perception of emotional stimuli: Brain mechanisms. Neuroscientist, 18(4), 386-398.

Morris, J. S., deBonis, M., \& Dolan, R. J. (2002). Human amygdala responses to fearful eyes. Neuroimage, 17(1), 214-222.

Morris, J. S., DeGelder, B., Weiskrantz, L., \& Dolan, R. J. (2001). Differential extrageniculostriate and amygdala responses to presentation of emotional faces in a cortically blind field. Brain, 124(Pt 6), 1241-1252.

Morris, J. S., Ohman, A., \& Dolan, R. J. (1998). Conscious and unconscious emotional learning in the human amygdala. Nature, 393(6684), 467-470.

Morris, J. S., Ohman, A., \& Dolan, R. J. (1999). A subcortical pathway to the right amygdala mediating "unseen" fear. Proceedings of the National Academy of Sciences, USA, 96(4), 1680-1685.

Nguyen, M. N., Matsumoto, J., Hori, E., Maior, R. S., Tomaz, C., Tran, A. H., et al (2014). Neuronal responses to face-like and facial stimuli in the monkey superior colliculus. Frontiers in Behavioral Neuroscience, 8, 85.

Overgaard, M. (2011). Visual experience and blindsight: A methodological review. Experimental Brain Research, 209(4), 473-479.

Panksepp, J. (2005). Affective consciousness: Core emotional feelings in animals and humans. Consciousness and Cognition, 14(1), 30-80.

Park, H. D., \& Tallon-Baudry, C. (2014). The neural subjective frame: From bodily signals to perceptual consciousness. Philosophical Transactions of the Royal Society of London. Series B, Biological sciences, 369(1641), 20130208.

Pasley, B. N., Mayes, L. C., \& Schultz, R. T. (2004). Subcortical discrimination of unperceived objects during binocular rivalry. Neuron, 42(1), 163-172.

Pegna, A. J., Khateb, A., Lazeyras, F., \& Seghier, M. L. (2005). Discriminating emotional faces without primary visual cortices involves the right amygdala. Nature Neuroscience, 8(1), 24-25.

Pessoa, L. (2005). To what extent are emotional visual stimuli processed without attention and awareness? Current Opinion in Neurobiology, 15(2), 188-196.

Pessoa, L., \& Adolphs, R. (2011). Emotion processing and the amygdala: From a 'low road' to 'many roads' of evaluating biological significance. Nature Reviews Neuroscience, 11(11), 773-783.

Pessoa, L., Japee, S., \& Ungerleider, L. G. (2005). Visual awareness and the detection of fearful faces. Emotion, 5(2), $243-247$.

Pöppel, E., Held, R., \& Frost, D. (1973). Residual visual function after brain wounds involving the central visual pathways in man. Nature, 243, 295-296.

Rafal, R. D., Koller, K., Bultitude, J., Mullins, P., Ward, R., Mitchell, A. S., et al. (submitted). Connectivity between the superior colliculus and the amygdala in humans and macaque monkeys: Virtual dissection with probabilistic DTI tractography.

Railo, H., Andersson, E., Kaasinen, V., Laine, T., \& Koivisto, M. (2014). Unlike in clinical blindsight patients, unconscious processing of chromatic information depends on early visual cortex in healthy humans. Brain Stimulation, 7(3), 415-420.

Rees, G., Kreiman, G., \& Koch, C. (2002). Neural correlates of consciousness in humans. Nature Reviews Neuroscience, 3(4), 261-270.

Rossion, B., de Gelder, B., Pourtois, G., Guerit, J. M., \& Weiskrantz, L. (2000). Early extrastriate activity without primary visual cortex in humans. Neuroscience Letters, 279(1), 25-28.

Sagaspe, P., Schwartz, S., \& Vuilleumier, P. (2011). Fear and stop: A role for the amygdala in motor inhibition by emotional signals. Neuroimage, 55, $1825-1835$.

Sahraie, A., Weiskrantz, L., Barbur, J. L., Simmons, A., Williams, S. C., \& Brammer, M. J. (1997). Pattern of neuronal activity associated with conscious and unconscious processing of visual signals. Proceedings of the National Academy of Sciences, USA, 94(17), 9406-9411.

Schmolesky, M. T., Wang, Y., Hanes, D. P., Thompson, K. G., Leutgeb, S., Schall, J. D., et al (1998). Signal timing across the macaque visual system. Journal of Neurophysiology, 79(6), 3272-3278.

Shi, C., \& Davis, M. (2001). Visual pathways involved in fear conditioning measured with fear-potentiated startle: Behavioral and anatomic studies. Journal of Neuroscience, 21(24), 9844-9855.

Silvanto, J., Walsh, V., \& Cowey, A. (2009). Abnormal functional connectivity between ipsilesional V5/MT+ and contralesional striate cortex (V1) in blindsight. Experimental Brain Research, 193(4), 645-650.

Tamietto, M., Castelli, L., Vighetti, S., Perozzo, P., Geminiani, G., Weiskrantz, L., et al (2009). Unseen facial and bodily expressions trigger fast emotional reactions. Proceedings of the National Academy of Sciences, USA, 106(42), 17661-17666.

Tamietto, M., Cauda, F., Celeghin, A., Diano, M., Costa, T., Cossa, F. M., et al (2015). Once you feel it, you see it: Insula and sensory-motor contribution to visual awareness for fearful bodies in parietal neglect. Cortex, 62, 56-72.

Tamietto, M., Cauda, F., Corazzini, L. L., Savazzi, S., Marzi, C. A., Goebel, R., et al (2010). Collicular vision guides nonconscious behavior. Journal of Cognitive Neuroscience, 22(5), 888-902.

Tamietto, M., \& de Gelder, B. (2008). Affective blindsight in the intact brain: Neural interhemispheric summation for unseen fearful expressions. Neuropsychologia, 46(3), 820-828.

Tamietto, M., \& de Gelder, B. (2010). Neural bases of the non-conscious perception of emotional signals. Nature Reviews Neuroscience, 11(10), 697-709.

Tamietto, M., Burra, N., Hervais-Adelman, A., Celeghin, A., Diano, M., Weiskrantz, L., et al. (in preparation). Affective blindsight depends on low spatial frequency and is mediated by a subcortical pathway to the amygdala.

Tamietto, M., Geminiani, G., \& De Gelder, B. (2006a). Inter-hemispheric cooperation for facial and bodily emotional expressions is independent of visual similarities between stimuli. Journal of Vision, 6(6), 1063a.

Tamietto, M., Latini Corazzini, L., de Gelder, B., \& Geminiani, G. (2006b). Functional asymmetry and interhemispheric cooperation in the perception of emotions from facial expressions. Experimental Brain Research, 171, 389-404.

Tamietto, M., Latini Corazzini, L., Pia, L., Zettin, M., Gionco, M., \& Geminiani, G. (2005). Effects of emotional face cueing on line bisection in neglect: A single case study. Neurocase, 11(6), 399-404.

Tamietto, M., Pullens, P., de Gelder, B., Weiskrantz, L., \& Goebel, R. (2012). Subcortical connections to human amygdala and changes following destruction of the visual cortex. Current Biology, 22(15), 1449-1455.

Tsuchiya, N., \& Adolphs, R. (2007). Emotion and consciousness. Trends in Cognitive Sciences, 11(4), 158-167.

Van den Stock, J., Tamietto, M., Hervais-Adelman, A., Pegna, A. J., \& de Gelder, B. (2013). Body recognition in a patient with bilateral primary visual cortex lesions correspondence. Biological Psychiatry.

Van den Stock, J., Tamietto, M., Sorger, B., Pichon, S., Grezes, J., \& de Gelder, B. (2011). Cortico-subcortical visual, somatosensory, and motor activations for perceiving dynamic whole-body emotional expressions with and without striate cortex (V1). Proceedings of the National Academy of Sciences, USA, 108(39), 16188-16193.

Van den Stock, J., Tamietto, M., Zhan, M., Heinecke, A., Hervais-Adelman, A., Legrand, L. B., et al (2014). Neural correlates of body and face perception following bilateral destruction of the primary visual cortices. Frontiers in Behavioral Neuroscience, 8, 30.

Vuilleumier, P. (2005). How brains beware: Neural mechanisms of emotional attention. Trends in Cognitive Sciences, 9(12), 585-594.

Vuilleumier, P., Armony, J. L., Driver, J., \& Dolan, R. J. (2003). Distinct spatial frequency sensitivities for processing faces and emotional expressions. Nature Neuroscience, 6(6), 624-631.

Ward, R., Calder, A. J., Parker, M., \& Arend, I. (2007). Emotion recognition following human pulvinar damage. Neuropsychologia, 45(8), $1973-1978$.

Ward, R., Danziger, S., \& Bamford, S. (2005). Response to visual threat following damage to the pulvinar. Current Biology, 15(6), 571-573. 
Weiskrantz, L. (1956). Behavioral changes associated with ablation of the amygdaloid complex in monkeys. Journal of Comparative and Physiological Psychology, 49(4), 381-391.

Weiskrantz, L. (1990). The Ferrier lecture, 1989. Outlooks for blindsight: Explicit methodologies for implicit processes. Proceedings of the Royal Society of London. Series B: Biological Sciences, 239(1296), 247-278.

Weiskrantz, L., Warrington, E. K., Sanders, M. D., \& Marshall, J. (1974). Visual capacity in the hemianopic field following a restricted occipital ablation. Brain, 97(4), 709-728.

Whalen, P. J., Kagan, J., Cook, R. G., Davis, F. C., Kim, H., Polis, S., et al (2004). Human amygdala responsivity to masked fearful eye whites. Science, 306(5704), 2061.

Whalen, P. J., Rauch, S. L., Etcoff, N. L., McInerney, S. C., Lee, M. B., \& Jenike, M. A. (1998). Masked presentations of emotional facial expressions modulate amygdala activity without explicit knowledge. The Journal of Neuroscience, 18(1), 411-418.

Williams, L. M. et al (2004b). Mapping the time course of nonconscious and conscious perception of fear: an integration of central and peripheral measures. Human Brain Mapping, 21, 64-74.

Williams, L. M., Das, P., Liddell, B. J., Kemp, A. H., Rennie, C. J., \& Gordon, E. (2006). Mode of functional connectivity in amygdala pathways dissociates level of awareness for signals of fear. The Journal of Neuroscience, 26(36), 9264-9271.

Williams, M. A., Morris, A. P., McGlone, F., Abbott, D. F., \& Mattingley, J. B. (2004a). Amygdala responses to fearful and happy facial expressions under conditions of binocular suppression. Journal of Neuroscience, 24, 2898-2904.

Winkielman, P., \& Berridge, K. C. (2004). Unconscious emotions. Current Directions in Psychological Science, 13(3), 120-123.

Zeman, A. (2001). Consciousness. Brain, 124, 1263-1289. 